\title{
Correlation of Neuro-cognition and Social Cognition of People with Schizophrenia
}

\author{
Jacky Y.H. Chan ${ }^{1^{*}}$, Serena S.W. $\mathrm{Ng}^{2}$, Ada W.Y. Lo ${ }^{1}$, Frances S.M. Chan ${ }^{1}$, Tony K.S. Leung ${ }^{1}$, Josephine W.M. Kwok ${ }^{1}$, Lawrence \\ W.L. Chow ${ }^{1}$, Vivian M.C. Tam ${ }^{1}$ and Cookie Y.M. Yiu ${ }^{1}$ \\ ${ }^{1}$ Department of Occupational Therapy, Kowloon Hospital, 147A Argyle St, Kowloon, Hong Kong \\ ${ }^{2}$ Community Rehabilitation Service Support Centre, Kowloon Hospital, 147A Argyle, Kowloon, Hong Kong
}

\section{Abstract}

Introduction: Apart from the traditional understanding on the relationship between neurocognitive deficits and functional impairment, there was a recent trend of investigation on the association of neurocognition and social cognition. Importance of neuro-cognitive functions and social functioning in sustaining supported employment among adults with schizophrenia was documented. However, there was little local documentation on the profile of persons with schizophrenia on these two areas.

Method: This is a prospective cohort analysis of forty-four in-patients admitted to a local rehabilitation setting diagnosed with schizophrenia and related disorders. The Matrics Consensus Cognitive Battery (MCCB) was chosen as standard measure.

Results: The results indicated that social cognitive and neurocognitive deficits were common among the subjects with schizophrenia. Social cognition $(M=30.18, S D+/-11.07)$, Verbal learning $(M=38.70$, $\mathrm{SD}=8.46)$ and Speed of processing $(\mathrm{M}=35.09, \mathrm{SD}=14.34)$ were the three most poorly performed cognitive domains. Social cognition was only significantly correlated with Visual learning $(\mathrm{r}(44)=.363$, $\mathrm{p}<.05$ ) but not the other neurocognitive domains.

Conclusion: MCCB is a reliable tool for routine assessment of cognitive functions for persons with schizophrenia. Further correlational studies were warranted including symptom severity and subjective measurementsto identify the linkage between these two constructs.

\section{Introduction}

Although engagement in productive activity or employment was the key factor in sustaining recovery for people with schizophrenia, Rosenheck et al. [1] stated that over $70 \%$ of the sampling subjects in their study were reported with no employment activity. Importance of neuro-cognitive functions and social functioning in sustaining supported employment among adults with severe mental illness (SMI) were reflected in a local study [2]. Deficiency in their productive living could lead to an enormous demand on social welfare and health maintenance. To understand the difficulties of the persons with schizophrenia especially in coping with productive work, investigations on neurocognitive functioning were often under the researchers' spotlights. Some of the studies even regarded neurocognitive impairmentas the core of the disorder [3] and was able to provide consistent explanation to functional impairments among people with schizophrenia [4].

Social cognition, which reflected one's ability in constructing mental representation about self and others [5], demonstrated stronger correlation with community functioning in comparing to neurocognitive functioning [6]. And it offered a unique perspective to quantify interpersonal difficulties in people with schizophrenia [7]. According to Lysaker et al. [8], the construct of social cognition consisted of four major components, including affect recognition, theory-of-mind, emotional intelligent and attribution style. Deficits in social cognition could be described as a divergence between actual and perceived social signal, which led to misunderstanding in social situations [9].

Studies on cognitive profiles of people with schizophrenia had indicated that speed of processing; together with social cognition best distinguished this group of individuals from a community sampling [10]. However, the relationship between neuro-cognition and social cognition remained debatable. Van Hooren et al. [11] conceptualized the deficits of social cognition and neuro-cognition in two separated pathways, named the "affective pathway" and "neurocognitive pathway" respectively, while the affective pathway accounted for their hypersensitivity in social situations and the neurocognitive pathway reflected structural deficits and other chronic courses of the disorder. Bell, Tsang, Greig and Bryson [12] had demonstrated how social cognition mediated the effect of neuro-cognition on community functioning in a three-factor model, proposing that impairment in neuro-cognition could lead to more problems with social cognition.

The purpose of this study aimed to document the first profile of the neuro-cognition and social cognition for a local sample of people with schizophrenia and explore the correlation among the two areas. We hope the results would facilitate further research in this area and fill the knowledge gap in local evidence.

\section{Objective}

1. To review the neurocognitive and social cognitive profiles of people with schizophrenia in a local rehabilitation setting.

2. To investigate the correlation between neuro-cognition and social cognition of people with schizophrenia or related illnesses.

"Corresponding Author: Mr. Jacky Y.H. Chan, Department of Occupational Therapy, Kowloon Hospital, 147A Argyle St, Kowloon, Hong Kong; E-mail: cyh825a@ha.org.hk

Citation: Chan JYH, Ng SSW, Lo AWY, Chan FSM, Leung TKS, et al. (2018) Correlation of Neuro-cognition and Social Cognition of People with Schizophrenia. Int J Psychol Behav Anal 4: 139. doi: https://doi.org/10.15344/2018/2455$3867 / 139$

Copyright: @ 2018 Chan et al. This is an open-access article distributed under the terms of the Creative Commons Attribution License, which permits unrestricted use, distribution, and reproduction in any medium, provided the original author and source are credited. 
Citation: Chan JYH, Ng SSW, Lo AWY, Chan FSM, Leung TKS, et al. (2018) Correlation of Neuro-cognition and Social Cognition of People with Schizophrenia. Int J Psychol Behav Anal 4: 139. doi: https://doi.org/10.15344/2018/2455-3867/139

Page 2 of 4

\begin{abstract}
Methodology
This is a prospective cohort analysis of patients admitted consecutively to occupational therapy services in a local hospital for mental health. Single point assessment performed by the case therapist with standardized tools on recording the neuro-cognitive and social cognitive states was performed, in addition to demographic and medical information.
\end{abstract}

\section{Measure}

Matrics Consensus Cognitive Battery (MCCB) was used in this study as the standard measure for neuro-cognitive functions, including social cognition. It is a standardized battery, tailoring for adults with schizophrenia and related illnesses. MCCB was a popular assessment tool among studies on schizophrenia [13,14] and it also demonstrated good test-retest reliability in researches among Chinese population [15]. MCCB included ten tests that measure seven cognitive domains, including: speed of processing, attention, working memory, verbal learning, visual learning, problem solving \& reasoning and social cognition. In order to create a common metrics between different tests, a standardization and co-norming process was conducted by Shi and colleagues [15] among 656 healthy volunteers in China. Domain scores were calculated by the MCCB computerscoring program and presented in term of $\mathrm{T}$-score and percentile so as to facilitate the interpretation of test result in clinical practices. The usual administration time of MCCB was around one to one and a half hours. Case therapists were trained as rater using MCCB.

\section{Subjects}

A cohort of individuals with an ICD-10 diagnosis of psychosis, schizophrenia or schizoaffective disorder referred for occupational therapy services during the period from February of 2015 to August of 2015 was recruited. All subjects were admitted to adult psychiatric wards with age limited from eighteen to sixty-five. People with neurological disorders or primary diagnosis of substance abuse were excluded. All assessments were conducted after the individuals were mentally stable.

\section{Data Analysis}

Descriptive statistics were performed on demographic data. In consideration of the significant effects from age, gender and education on normative data [15], we adopted the packaged computation "Age, gender and education corrected" of MCCB for calculation in order to minimize the influences from demographic variances. Kendall rank correlations were performed for correlation analysis across the seven domain T-scores in MCCB in view of the small sampling size. Statistical package for the social science (SPSS) version 22 was used.

\section{Result}

\section{Demographics}

Forty-four subjects completed the data collection. Thirty-three (75\%) of them were diagnosed with schizophrenia, eight of them with paranoid schizophrenia and three of them with other psychotic disorders, including psychosis and simple type schizophrenia. Average sample age is $39.36(M=39.36, S . D .=13.26)$. Twenty-two $(50 \%)$ of them were female. Their average years of education were 11.84
$(\mathrm{M}=11.84, \mathrm{SD}=3.29)$. The average years of onset were 9.43 years $(M=$ $9.43, S D=10.75)$ and the number of previous psychiatric admissions in average was $2.86(M=2.86, S D=4.23)$.

\section{Profile of neuro-cognition and social cognition}

For the 44 subjects, with age, gender and education corrected, the mean T-scores of MCCB domain tests presented in Table 1, indicated the presence of impairments (below one standard deviation from mean T-score) across six domains in MCCB, except "Working memory" $(M=47.29, S D=12.34)$. The domain scores of "Social cognition" $(M=30.18, S D=11.07)$, "Verbal learning" $(M=38.70, \mathrm{SD}$ $=8.46)$ and "Speed of processing" $(M=35.09, S D=14.34)$ were the three most poorly performed cognitive domains.

\begin{tabular}{|l|l|l|l|l|}
\hline & & $\mathrm{N}$ & $\begin{array}{l}\text { Mean } \\
\text { T-score }\end{array}$ & $\begin{array}{l}\text { Standard } \\
\text { Deviation }\end{array}$ \\
\hline 1. & Speed of processing & 44 & 35.09 & 14.34 \\
\hline 2. & Attention & 44 & 40.11 & 13.20 \\
\hline 3. & Working memory & 44 & 47.29 & 12.34 \\
\hline 4. & Verbal learning & 44 & 38.70 & 8.46 \\
\hline 5. & Visual learning & 44 & 39.80 & 14.97 \\
\hline 6. & Reasoning and problem solving & 44 & 40.64 & 9.36 \\
\hline 7. & Social cognition & 44 & 30.18 & 11.07 \\
\hline
\end{tabular}

Table 1: T-scores of the Seven Domains.

In this study, MCCB was firstly presented with documented results for persons with schizophrenia from a local hospital. It was regarded as a reliable tool from the results shown in our sample, with Cronbach's Alpha of 0.787 across 7 domain items. Inter-item correlation distinguished 'social cognition' from others which specifically poor from 0.075 in "social cognition vs attention", 0.095 in "social cognition vs working memory" and 0.099 in "social cognition vs reasoning / problem solving"; while other items ranges from 0.230 to 0.617 . Cochrane's Test for Between-items Means Square of whole MCCB was $59.746(\mathrm{p}<0.001)$.

The result of Kendall rank correlation test showed similar findings with significant correlations $(\mathrm{p}<0.05)$ shown among all the domain scores except social cognition. 'Social cognition' was only correlated with the domain score of 'Visual learning' $(\mathrm{r}(44)=0.363, \mathrm{p}<0.05)$ (Table 2, Figure 1).

\section{Discussion}

This is the first local series on the cognitive profiles of people with schizophrenia, using Matrics Consensus Cognitive Battery (MCCB) as a measurement tool and was found reliable. The results showed that neurocognitive deficits were common among the subjects with schizophrenia in our study, particularly on social cognition, verbal learning and speed of processing. The study of Kern et al. [10] on 176 people with schizophrenia or schizoaffective disorder by MCCB had stated that speed of processing; working memory and social cognition were the three most impaired domains, which echoed with our findings. Concerning about the impairment of speed of processing across multiple studies, Knowles, David and Reichenberg [16] had stated that it could be the largest single cognitive impairment in schizophrenia, while Bachman et al. [17] suggested that neurological vulnerability, failure to utilize relational memory and dosage of particular antipsychotic medication account for this impairment. 
Citation: Chan JYH, Ng SSW, Lo AWY, Chan FSM, Leung TKS, et al. (2018) Correlation of Neuro-cognition and Social Cognition of People with Schizophrenia. Int J Psychol Behav Anal 4: 139. doi: https://doi.org/10.15344/2018/2455-3867/139

Page 3 of 4

\begin{tabular}{|c|c|c|c|c|c|c|c|}
\hline & 1 & 2 & 3 & 4 & 5 & 6 & 7 \\
\hline 1. Speed of processing & --- & $.360^{* *}$ & .181 & $.406^{* *}$ & $.401^{* *}$ & $.478^{\star *}$ & .114 \\
\hline 2. Attention & $.360^{*}$ & --- & $.286^{* *}$ & $.335^{\star *}$ & $.313^{* *}$ & $.349^{* *}$ & .068 \\
\hline 3. Working memory & .181 & $.286^{* *}$ & --- & $.259^{*}$ & .126 & .193 & -.026 \\
\hline 4. Verbal Learning & $.406^{* *}$ & $.335^{\star *}$ & $.259^{*}$ & --- & $.333^{\star *}$ & $.283^{\star *}$ & .189 \\
\hline 7. Social cognition & .114 & .068 & -.026 & .189 & $.236^{*}$ & .099 & --- \\
\hline
\end{tabular}

Table 2: Correlations Among the Seven Domain Scores

** Correlation is significant at the 0.01 level (2-tailed)

* Correlation is significant at the 0.05 level (2-tailed)

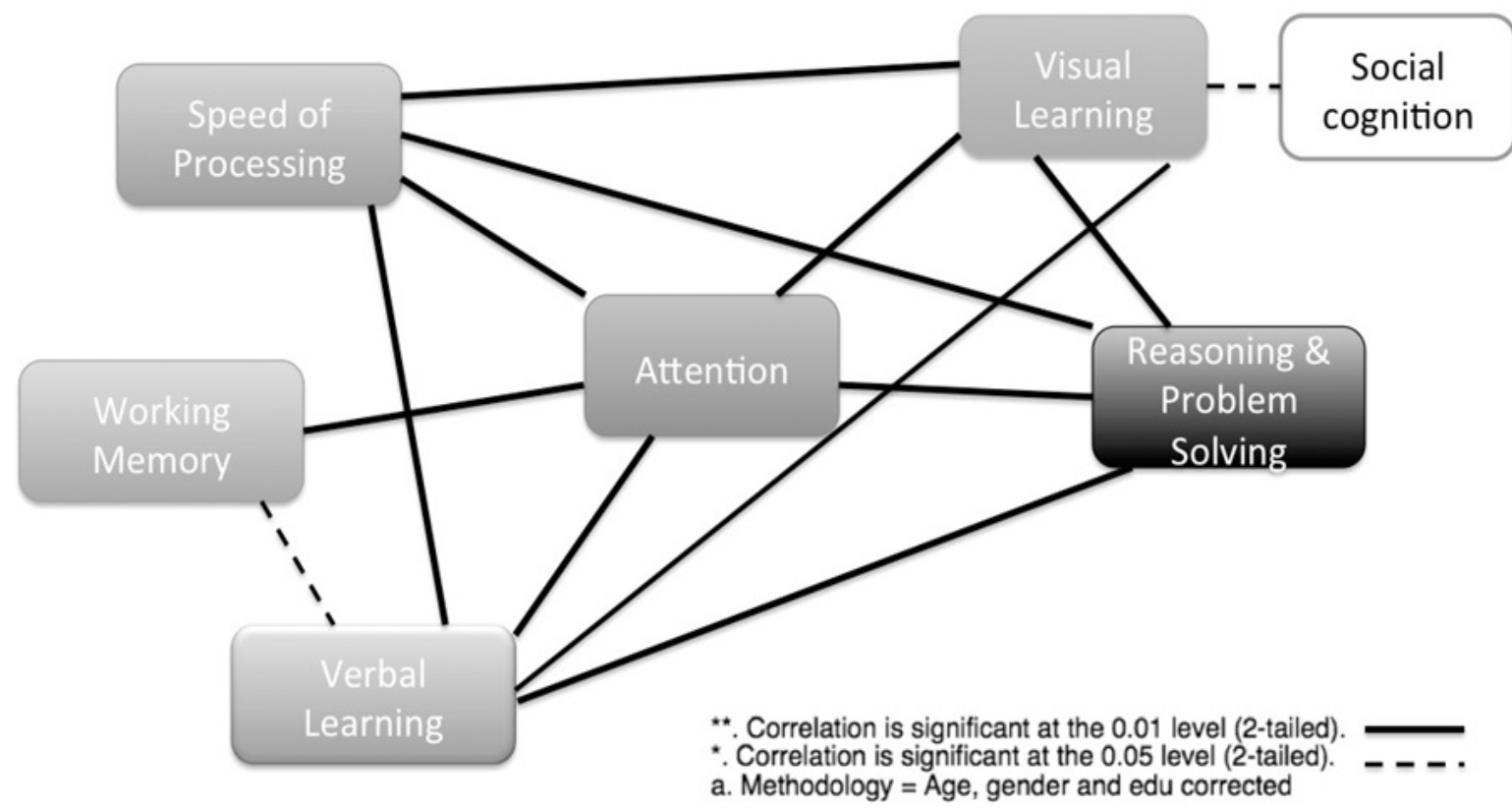

Figure 1: Correlations Among the Severn Domain Scores.

Beside, McCleery et al. [18] stated that functioning of working memory and social cognition were relatively preserved among people with first episode schizophrenia when compared to people with chronic schizophrenia. Thus, it was not surprised that our current sample, a relatively advanced illness profile (Year of onset, $M=9.43$, $\mathrm{SD}=10.75$ )demonstrated a declining performance in social cognition along the progression of disorder.

Unlike other neurocognitive domains which are inter-correlated in a significant manner, social cognition was only correlated significantly with visual learning $(r(44)=.363, p<.05)$. Social cognition, under the construct of $\mathrm{MCCB}$, might reflect different characteristics than the remaining neuro-cognitive areas. The results of correlational analysis of our study was similar to the idea suggested by Van Hooren et al. [11] that neuro-cognition and social cognition were nonoverlapping areas of vulnerability in people with psychosis. Evidence on correlation between social cognition and visual learning was still limited, although it had been proved that one's gaze cueing and visual attention could influence on perception of others [19]. Further research on the relationship among these two areas may shed light on design of interventions for persons with schizophrenia in enhancing their productive activities in social context.

\section{Limitation}

Small sample size was the obvious limitation of this project, which restricted our choice in statistical analysis and generalization. The wide age range of subjects also raised our concern, as aging was a crucial factor, leading to changes in neurocognitive functioning [20]. The social cognitive domain of MCCB was measured by MayerSalovey-Caruso Emotional Intelligence Test (MSCEIT), focused on measurement on emotional intelligence. The properties of affect recognition, theory of mind and attribution style in social cognition remained unexamined, which also limited our choice in correlational testing. Finally, previous studies had suggested that correlational analysis between neuro-cognition and social cognition often completed in a three factors model. Without capturing the severity of symptoms of the subjects, our opportunity to examine how one's mental condition moderates social cognitive and neurocognitive performances was limited. 
Citation: Chan JYH, Ng SSW, Lo AWY, Chan FSM, Leung TKS, et al. (2018) Correlation of Neuro-cognition and Social Cognition of People with Schizophrenia. Int J Psychol Behav Anal 4: 139. doi: https://doi.org/10.15344/2018/2455-3867/139

Page 4 of 4

\section{Conclusion}

This study has demonstrated that impairment in social cognition was common among people with schizophrenia. However, the correlation between neuro-cognition and social cognition remained debatable. It was postulated that the inclusion of symptomatic or subjective measurement in social situation might help to clarify the linkage between these two constructs. Besides, detailed investigation on constructing components of social cognition could open more opportunity for correlation analysis study in future.

\section{Competing Interests}

The authors declare that they have no competing interests.

\section{References}

1. Rosenheck R, Leslie D, Keefe R, McEvoy J, Swartz M, et al. (2006) Barriers to employment for people with schizophrenia. Am J Psychiatry 163: $411-417$.

2. S Ng, D Lak, S, Lee, P Ng (2015) Concurrent validation of the success profile of patients with SMI for supported employment as shop sales in NGO. East Asian Archives of Psychiatry 25: 21-28.

3. Elvevag B, Goldberg TE (2000) Cognitive impairment in schizophrenia is the core of the disorder. Crit Rev Neurobiol 14: 1-21.

4. McGurk SR, Mueser KT, Harvey PD, LaPuglia R, Marder J (2003) Cognitive and symptom predictors of work outcomes for clients with schizophrenia in supported employment. Psychiatr Ser 54: 1129-1135.

5. Sergi MJ, Rassovsky Y, Widmark C, Reist C, Erhart S, et al. (2007) Social cognition in schizophrenia: relationships with neurocognition and negative symptoms. Schizophr Res 90: 316-324.

6. Fett AKJ, Viechtbauer $\mathrm{W}$, Dominguez MD, Penn DL, van Os J, et al (2011) The relationship between neurocognition and social cognition with functional outcomes in schizophrenia: a meta-analysis. Neurosci Biobehav Rev 35: 573-588.

7. Henry JD, Cowan DG, Lee T, Sachdev PS (2015) Recent trends in testing social cognition. Curr Opin Psychiatry 28: 133-140.

8. Lysaker PH, Gumley A, Luedtke B, Buck KD, Ringer JM, et al. (2013) Social cognition and metacognition in schizophrenia: evidence of their independence and linkage with outcomes. Acta Psychiatr Scand 127: 239247.

9. Wiltshire TJ, Lobato EJ, McConnell DS, Fiore SM (2014) Prospects fo direct social perception: a multi-theoretical integration to further the science of social cognition. Front Hum Neurosci 8: 1007

10. Kern RS, Gold JM, Dickinson D, Green MF, Nuechterlein KH, et al. (2011) The MCCB impairment profile for schizophrenia outpatients: results from the MATRICS psychometric and standardization study. Schizophr Res 126: 124-131.

11. Van Hooren S, Versmissen D, Janssen I, Myin-Germeys I, à Campo J, et al. (2008) Social cognition and neurocognition as independent domains in psychosis. Schizophr Res 103: 257-265.

12. Bell M, Tsang HW, Greig TC, Bryson GJ (2009) Neurocognition, social cognition, perceived social discomfort, and vocational outcomes in schizophrenia. Schizophr Bull 35:738-747

13. Lin $\mathrm{CH}$, Huang CL, Chang YC, Chen PW, Lin CY, et al. (2013) Clinical symptoms, mainly negative symptoms, mediate the influence of neurocognition and social cognition on functional outcome of schizophrenia. Schizophrenia research 146: 231-237.

14. Linke M, Jankowski KS, Ciołkiewicz A, Jędrasik-Styła M, Parnowska D, et al. (2015) Age or age at onset? Which of them really matters for neuro and social cognition in schizophrenia? Psychiatry Research 225: 197-201.

15. Shi C, Kang L, Yao S, Ma Y, Li T, et al. (2015) The MATRICS Consensus Cognitive Battery (MCCB): Co-norming and standardization in China. Schizophr Res 169: 109-115.

16. Knowles EE, David AS, Reichenberg A (2010) Processing speed deficits in schizophrenia: reexamining the evidence. Am J Psychiatry 167: 828-835.

Int J Psychol Behav Anal

ISSN: 2456-3501
17. Bachman P, Reichenberg A, Rice P, Woolsey M, Chaves O, et al. (2010) Deconstructing processing speed deficits in schizophrenia: application of a parametric digit symbol coding test. Schizophr Res 118: 6-11.

18. McCleery A, Ventura J, Kern RS, Subotnik KL, Gretchen-Doorly D, et al (2014) Cognitive functioning in first-episode schizophrenia: MATRICS Consensus Cognitive Battery (MCCB) Profile of Impairment. Schizophr Res 157: 33-39.

19. Frischen A, Bayliss AP, Tipper SP (2007) Gaze cueing of attention: visual attention, social cognition, and individual differences. Psychol bull 133:694.

20. Wang Y, Roberts DL, Xu B, Cao R, Yan M, et al. (2013) Social cognition and interaction training for patients with stable schizophrenia in Chinese community settings. Psychiatry Res 210: 751-755. 